\title{
Dynamical properties of the conformally coupled flat FRW model
}

\author{
M. A. Castagnino \\ Instituto de Astronomía y Física del Espacio \\ Casilla de correo 67, sucursal 28 \\ 1428 , Buenos Aires, Argentina. \\ H. Giacomini \\ Laboratoire de Mathématiques et Physique Théorique- UPRES A 6083 \\ Faculté des Sciences et Techniques \\ Université de Tours-Parc de Grandmont \\ 37200 Tours, France \\ and FCBF-Area de Física, UNR \\ Suipacha 531, 2000 Rosario, Argentina. \\ L. Lara \\ Depto. de Física, FCEIA, UNR \\ Avda Pellegrini 250, 2000 Rosario, Argentina. \\ lplara@arnet.com.ar
}

\begin{abstract}
In this paper we study the dynamical behaviour of a simple cosmological model defined by a spatially flat Robertson-Walker geometry, conformally coupled with a massive scalar field. We determine a Lyapunov-like function for the non-linear evolution equations. From this function we prove that all the stationary solutions are unstable. We also show that all initial conditions, different from the stationary points, originate an expanding universe in the asymptotic regime, with a scale parameter $a(t)$ that goes to infinity and the scalar field $\phi(t)$ that goes to zero in an oscillatory way . We also find two asymptotic solutions, valid for sufficiently large values of time. These solutions correspond to a radiation dominated phase and to a matter dominated phase, respectively.
\end{abstract}

\section{INTRODUCTION}

In this work we briefly analyze some new properties of an oversimplified cosmological model: a spatially flat Robertson-Walker geometry, conformally coupled with a massive scalar field, which has been studied in many papers [1]. The universe is well described by spatially flat $(k=0)$ cosmological models, which are also compulsory if we use inflationary theories, and an inflation field is usually included in these models. Moreover, the conformal coupling is necessary if we want to satisfy the equivalence principle [2]. Hence, the studied oversimplified structure is necessarily contained in the universe, albeit complemented by much more detailed features. Since this simplified stru

cture by itself implies several of the most important cosmological properties we believe that the results of this letter are of interest.

These properties are:

a.- It expands to infinity for all non-stationary initial conditions.

b.- It contains a Lyapunov-like 1 function $F$ with a positive time derivative and therefore is essentially time asymmetric.

\footnotetext{
${ }^{1} \mathrm{~A}$ Lyapunov function is an evergrowing function of $t$ that vanishes at a critical point and it is different from zero in a neighborhood of this point. Our function is not a proper Lyapunov function because it is not of definite sign.
} 
c.- The final phase of the universe evolution is either radiation or matter dominated.

d.- The scalar field $\phi(t)$ goes to zero in an oscillatory way in the asymptotic regime.

\section{THE MODEL}

Let us consider a flat Robertson-Walker geometry with scale factor $a(t)$ conformally coupled with a neutral massive scalar field $\phi(t)$ of mass $m$. The hamiltonian reads [3]:

$$
\mathcal{H}=\frac{1}{2}\left(\dot{\phi}^{2}-\dot{a}^{2}\right)+\frac{m^{2}}{2} a^{2} \phi^{2}=\frac{1}{m^{2}} k
$$

where

$$
k=\frac{1}{2}\left(\dot{x}^{2}-\dot{y}^{2}\right)+\frac{1}{2} x^{2} y^{2}
$$

$x=m \phi, y=m a, p_{x}=\dot{x}, p_{y}=\dot{y}$ and the dots indicate derivative with respect to the conformal time $t$, defined by the relation $d t_{p}=a(t) d t$, where $t_{p}$ is the commoving or proper time. The field equations read:

$$
\ddot{x}=-x y^{2}, \quad \ddot{y}=y x^{2}
$$

and the Einstein constraint is $\mathcal{H}=0$. This condition expresses the Hamiltonian constraint of general relativity. The fixed points are located at:

$$
\begin{aligned}
& x \text { arbitrary, } \quad y=p_{x}=p_{y}=0 \\
& y \text { arbitrary, } \quad x=p_{x}=p_{y}=0
\end{aligned}
$$

These fixed points are stationary solutions of the system.

\section{MAIN DYNAMICAL PROPERTIES OF THE MODEL}

Let us define the function:

$$
F(y, \dot{y})=y \dot{y}
$$

Taking the time derivative of this function along an orbit of the system and using the second equation (3) we obtain:

$$
\frac{d F}{d t}=\dot{y}^{2}+x^{2} y^{2} \geqq 0
$$

Hence, function $F$ is a monotonous growing function of $t$. Then, we have

$$
\lim _{t \rightarrow+\infty} F(y(t), \dot{y}(t))=+\infty
$$

for an arbitrary non-stationary initial condition. The case $\lim _{t \rightarrow+\infty} F(y(t), \dot{y}(t))=C$, where $C$ is a constant, is excluded since for great values of $t$ we have $y(t) \cong \pm \sqrt{2 C t+C_{0}}$ and the second equation (3) can not be satisfied for real values of $x$. Here, $C_{0}$ is an integration constant that appears when we solve the differential equation $\left.y(t) \dot{y}(t)\right) \cong C$. We conclude that $\lim _{t \rightarrow+\infty} y(t)= \pm \infty$, i.e. all the orbits in phase space $\left(x, y, p_{x}, p_{y}\right)$ go to infinity. It is clear that eq. (5) forbids the existence of periodic solutions. If a periodic solution $x(t), y(t)$ of period $T$ exists, integrating both sides of eq. (5) along this solution we obtain zero in the left-hand side and a positive number in the right-hand side and we have a contradiction. All initial conditions different from the fixed points yield an universe evolution that end in and expanding phase.

Moreover, from this fact we see that all the fixed points are unstable. We also conclude that the system has no chaotic behaviour because all the orbits different of the fixed points are not bounded (see [4]).

Let us study now the asymptotic behaviour of the scalar field. For great values of $t$ the function $y(t)$ goes to $\pm \infty$ in a monotonous way, and the product $y \dot{y}$ is positive. Then, the function $G(y, \dot{y})=\frac{\dot{y}}{y}$ is well defined and positive in 
the asymptotic regime. Taking the time derivative of $G$ along an orbit of the system and using the second eq. (3) we obtain

$$
\dot{G}=\frac{\ddot{y}}{y}-\frac{\dot{y}^{2}}{y^{2}}=\frac{y^{2} x^{2}-\dot{y}^{2}}{y^{2}}
$$

The constraint $\mathcal{H}=0$ gives

$$
\dot{y}^{2}=\dot{x}^{2}+x^{2} y^{2}
$$

Replacing this expression of $\dot{y}^{2}$ in eq. (7) we obtain

$$
\dot{G}=-\frac{\dot{x}^{2}}{y^{2}} \leq 0
$$

Since the function $G$ is positive and always decreasing we have

$$
\lim _{t \rightarrow+\infty} G=\lim _{t \rightarrow+\infty} \frac{\dot{y}}{y}=k
$$

where $k$ is a non-negative constant. Since function $G$ tends to a constant value in a monotonous way, its first derivative must tends to zero when $t \rightarrow+\infty$, i.e.

$$
\lim _{t \rightarrow+\infty} \dot{G}=\lim _{t \rightarrow+\infty}-\frac{\dot{x}^{2}}{y^{2}}=0
$$

Equation (8) can be written as follows

$$
\frac{\dot{y}^{2}}{y^{2}}=\frac{\dot{x}^{2}}{y^{2}}+x^{2}
$$

Taking the limit when $t \rightarrow+\infty$ in both sides of this equality and using eqs. (10) and (11), we obtain

$$
\lim _{t \rightarrow+\infty} x^{2}=k^{2}
$$

We will now show that the constant $k$ is zero. From eq. (13) we have that $\lim _{t \rightarrow+\infty} x= \pm k$. Let us consider a first case where the limit is positive. For sufficiently great values of $t, x$ must approach the value $+k$ in a monotonous way. In fact, small oscillatory behaviour with decreasing amplitude around the value $+k$ is impossible, since such type of behaviour implies a sequence of changes of sign of the second derivative of $x$. From the first equation (3) we see that it is impossible because the function $x$ does not change sign.

But, if the function $x$ approaches the value $+k$ in a monotonous way, its first and second derivatives must go to zero when $t \rightarrow+\infty$. Since $\lim _{t \rightarrow+\infty} y^{2}=+\infty$, we see from the first eq. (3) that $\ddot{x}$ can not go to zero. The analysis of the case where $x$ approaches the value $-k$ is analogous. Then, we conclude that the constant $k$ must be zero, i.e.

$$
\lim _{t \rightarrow+\infty} x=0
$$

We have proved that $x$ can not approach the value $\pm k$ in a monotonous way. The analysis is independent of the value of $k$. Then, we conclude that $x$ has an oscillatory behaviour with a decreasing amplitude when $t \rightarrow+\infty$. This oscillatory behaviour is not possible for $k \neq 0$ but it becomes possible in the case $k=0$ because $x(t)$ can change sign.

To summarise, we have proved that the scalar field $\phi=\frac{x}{m}$ goes to zero in a oscillatory way, the factor scale $a^{2}$ goes to infinity in a monotonous way, and $\frac{\dot{a}}{a}$ goes to zero when $t \rightarrow+\infty$ (eq.10 with $k=0$ ). 


\section{ASYMPTOTIC SOLUTIONS}

For $t>>1$ we can obtain explicit asymptotic solutions. Instead of system (3) we will consider the system:

$$
\ddot{x}=-x y^{2}, \quad \mathcal{H}=0
$$

because deriving the second equation with respect to $t$ and using the first one we obtain $\ddot{y}=y x^{2}$. System (15) automatically satisfies the constraint $\mathcal{H}=0$, while the system (3) has solutions that do not satisfy this constraint.

Inspired by exhaustive numerical calculations we have been able to determine two different asymptotic solutions for $t>>1$, namely:

$$
x(t) \sim \frac{1}{t} \sin \left(\frac{1}{2} t^{2}\right), \quad y(t) \sim t
$$

and

$$
x(t) \sim \frac{2}{t} \sin \left(\frac{1}{3} t^{3}\right), \quad y(t) \sim t^{2}
$$

Introducing these expressions in system (15) and taking $t>>1$, it can easily be verified that (16) and (17) are asymptotic solutions. Expressions (16) and (17) agree with the general results of section III.

Expressing these asymptotic solutions in terms of commoving time $t_{p}$ we conclude that:

a.- The final phase of solution (16) is radiation dominated (since $y\left(t_{P}\right) \sim t_{P}^{\frac{1}{2}}$ ).

b.- The final phase of solution (17) is matter dominated (since $y\left(t_{P}\right) \sim t_{P}^{\frac{2}{3}}$ ).

We have proved that (16) and (17) are asymptotic solutions but this does not imply that they are the unique solutions for $t \gg 1$. For a large number of initial conditions chosen at random, numerical computations show that the associated asymptotic solution is given by (17), i.e. a matter dominated evolution.

We have performed our analysis in the conformal time $t$. In general, to translate the results to the commoving time $t_{p}$ is not an easy matter (in some cases it is practically impossible) when the scale factor $a(t)$ is not monotonous. In our case, for sufficiently large values of $t, a(t)$ is monotonous and the asymptotic behaviour in the variable $t$ can be easily translated to the commoving time $t_{p}$. If we choose physical initial conditions in such a way that $a(t=0) \dot{a}(t=0)>0$, it is easy to show that $a(t)$ is a monotonous function of $t$. Then, for such initial conditions all the results obtained in terms of $t$ can be easily translated in terms of commoving time $t_{p}$, for arbitrary values of $t$. For more general cosmological models where the scale factor does not has a monotonous behaviour, the dynamical analysis in terms of conformal time $t$ is not justified. In fact, it is clearly wrong from the point of view of dynamical system theory.

\section{CONCLUSIONS}

In spite of the simplified nature of our cosmological model, its dynamical evolution is controlled by highly non-linear equations. Nevertheless, we have been able to obtain, in a rigorous way, the most relevant dynamical properties of the model: the instability of all the stationary solutions, the expansive nature of the evolution, the fact that the scalar field $\phi(t)$ goes to zero in oscillatory way and explicit expressions for the asymptotic solutions, giving the two possible phases of the universe.

Under the time inversion $t \rightarrow-t$ the hamiltonian (2), the field equations (3) and the constraint $\mathcal{H}=0$ remain invariant, so the system is trivially time-symmetric. Nevertheless, if the motion begins at a finite point with a finite value of $F$ (and this would be the case for the real universe that began very small and with high temperature in a quantum phase), the motion always goes to infinity. Then, it is time asymmetric since its initial and final states are different. Of course, the inverse motion is also a solution of the evolution equations. But the motion towards infinity is usually expanding and with creation of matter-field energy, and therefore corresponds to what we see in the observable universe.

[1] Futamase, T., Rothman, T., Matzner, R., Behaviour of chaotic inflation in anisotropic cosmologies with nonminimal coupling. Phys. Rev. D39, 2, 405 (1989). 
Futanase, T., Maeda, K., Chaotic inflationary scenario of the Universe with nonminimally coupled inflation field. Phys. Rev. D39, 2, 399 (1989).

Demianski, M., et al. Scalar field, nonminimal coupling and cosmology. Phys. Rev. D44, 10, 3136 (1991).

Peebles, P., Principles of Physical Cosmology, Princeton Univerty Press (1993).

Kolb, E. W., Turner, M. S., The Early Universe (Addison-Wesley, Reading, Massachusets)(1990).

[2] Sonego, S., Faraoni, V., Class. Quant. Grav. 10, 1185 (1993).

[3] Halliwell, J., Hawking, S, Origin of structure in the Universe, Phys. Rev. D31, 1777 (1985).

Castagnino, M., Gunzig, E. , Nardone P., On Quantum Gravity for Homogeneous Pure Radiation Universes, in Quantum Mechanics in Curved Space-Time (NATO, Erice, 1989, J. Audretsch, V. de Sabbata Eds.), pag. 403-431. Plennum Press, 1990.

Castagnino M., Gunzig E., Nardone P., The problem of the wave function of the universe in the radiation dominated case, Proceeding V Moscow Quantum Gravity Seminar 1990, page 600. E, Markov et al. Eds. World Scientific, Singapore (1991).

[4] Rocha Filho, T., Figueiredo, A., et al, Self-Consistent Scalar Field Cosmologies: Analytic Solutions. To be published.

Gunzig, E., Faraoni, V., et al, The dynamical system approach to scalar field cosmology. To be published. 